\title{
EFFICACITÉ D'UN EXUTOIRE DE DÉVALAISON POUR SMOLTS DE SAUMON ATLANTIQUE (SALMO SALAR L.) ET COMPORTEMENT DES POISSONS AU NIVEAU DE L'AMÉNAGEMENT HYDROÉLECTRIQUE DE BEDOUS SUR LE GAVE D'ASPE ÉTUDIÉS PAR LA TECHNIQUE DE MARQUAGE-RECAPTURE ET PAR RADIOTÉLÉMÉTRIE.
}

\author{
M. CHANSEAU (1), M. LARINIER (1) et F. TRAVADE (2)
}

(1) CSP-CEMAGREF-GHAAPPE, Institut de Mécanique des Fluides, Avenue du Professeur Camille Soula, 31400 Toulouse, France.

(2) Électricité de France - Études et Recherches, 6 Quai Watier, 78401 Chatou Cedex, France.

\section{RÉSUMÉ}

Deux expérimentations ont été conduites en 1995 et 1998 au niveau de la prise d'eau de l'aménagement hydroélectrique de Bedous sur le Gave d'Aspe afin de tester l'efficacité d'un exutoire de dévalaison pour smolts de saumon atlantique (Salmo salar L.).

Le dispositif de dévalaison est situé en rive droite à proximité immédiate du plan des grilles. Le débit moyen alimentant l'exutoire a varié au cours de l'étude de $0,4 \mathrm{~m}^{3} / \mathrm{s}$ à $1,2 \mathrm{~m}^{3} / \mathrm{s}$ ce qui correspond à $1,6 \%$ à $4,3 \%$ du débit turbiné. L'efficacité du dispositif a été évaluée grâce à la technique de marquage-recapture. Le comportement des smolts dans le canal d'amenée a été suivi par radiotélémétrie.

L'efficacité de l'exutoire (moyenne : $17 \%$ ) s'est révélée très faible en 1995 à̀ cause de conditions hydrauliques défavorables. Un mur déflecteur a été mis en place en 1997 afin de modifier l'écoulement dans le canal et améliorer le guidage des poissons vers l'entrée du dispositif de dévalaison. L'efficacité de l'exutoire est passée à $55 \%$. Le comportement des poissons à l'amont immédiat des grilles de prise d'eau est très dépendant des conditions hydrodynamiques dans le canal d'amenée. L'efficacité de l'exutoire semble croître avec la longueur des poissons, la proportion des classes de taille inférieures à $19 \mathrm{~cm}$ étant plus faible sur les poissons piégés que sur les poissons lâchés. Cette sélection est très certainement liée à l'espacement relativement important des barreaux des grilles de la prise d'eau $(30 \mathrm{~mm})$ qui malgré le courant tangentiel au plan de grilles demeurent plus perméables aux individus les plus petits. L'éclairage de l'entrée de l'exutoire ne paraît pas avoir d'influence sur les déplacements des poissons radiomarqués dans le canal d'amenée, le facteur hydraulique étant prépondérant. II modifie cependant les rythmes de passage des poissons par l'exutoire.

Mots-clés : dévalaison, aménagement hydroélectrique, exutoire de surface, marquage-recapture, radiotélémétrie, smolt, Salmo salar, comportement, hydrodynamique, lumière. 


\section{EFFICIENCY OF A DOWNSTREAM BYPASS AS ESTIMATED BY THE MARK-RECAPTURE TECHNIQUE AND BEHAVIOUR OF ATLANTIC SALMON (SALMO SALAR L.) SMOLTS AT THE BEDOUS WATER INTAKE ON THE ASPE RIVER (FRANCE) MONITORED BY RADIOTELEMETRY.}

\section{ABSTRACT}

Two experiments were conducted at the Bedous water intake on the Aspe River in 1995 and 1998 to test the efficiency of a downstream bypass for salmon smolts (Salmo salar L.).

The surface bypass was located on the right bank a little way upstream from the trashrack. During the experiment period, the discharge into the bypass varied from $0.4 \mathrm{~m}^{3} / \mathrm{s}$ to $1.2 \mathrm{~m}^{3} / \mathrm{s}$, representing from $1.6 \%$ to $4.3 \%$ of the turbine discharge. The efficiency of the device was evaluated using the mark-recapture technique. The behaviour of smolts in the intake canal was studied using radiotelemetry.

In 1995 the bypass efficiency was very low (with a success rate of $17 \%$ ), due mainly to hydraulic conditions. A training wall was built in 1997 to reverse the flow pattern in the canal and to better guide the fish to the water intake of the bypass. The mean efficiency of the bypass was thus improved with $55 \%$ of the smolts being recaptured in the trap in 1998. The efficiency of the device and the smolt behaviour were directly affected by the turbine operation and the hydraulic conditions in the intake channel. It appeared that a smaller proportion of smolts of less than $19 \mathrm{~cm}$ in length were recaptured in the trap and that in spite of the tangential component of the velocity which created a louver effect, size selection was due to the spacing between the bars of the trashracks $(30 \mathrm{~mm})$. A light placed near the bypass entrance appeared to have no effect on the movement patterns of the smolts with the hydraulic factor being preponderant. However, the light did affect the rhythm of capture in the trap as more fish were captured when it was turned off.

Key-words : downstream migration, hydroelectric power plant, surface bypass, mark-recapture, radiotelemetry, smolt, Salmo salar, behaviour, hydrodynamics, light.

\section{INTRODUCTION}

Depuis une vingtaine d'années, de nombreux programmes de restauration et de protection des poissons migrateurs ont été mis en place en France. L'une des causes essentielles de la diminution, voire de la disparition du saumon atlantique (Salmo salar L.), et des autres espèces migratrices sur les cours d'eau français, a été l'édification de barrages notamment pour la production d'énergie hydraulique (PORCHER et TRAVADE, 1992). Dans un premier temps, ces programmes ont eu pour objet de rendre les obstacles perméables à la migration anadrome afin que les poissons puissent atteindre les meilleures zones de frayère. Plus récemment, une attention particulière a été portée aux problèmes liés au franchissement des aménagements hydroélectriques par les juvéniles, la dévalaison ne se faisant pas sans dommages lorsque les migrateurs sont entraînés dans les prises d'eau des centrales hydroélectriques (LARINIER et DARTIGUELONGUE, 1989). C'est le cas en particulier de l'aménagement de Bedous-Asasp, où, la mortalité potentielle sur les smolts transitant dans les turbines étant supérieure à $50 \%$, il était impératif d'installer un dispositif efficace pour la dévalaison.

Un des moyens les plus simples et certainement les moins coûteux à mettre en oeuvre afin d'éviter le transit des poissons et notamment des smolts de saumon par les 
turbines est un exutoire de surface convenablement placé à proximité des grilles de prise d'eau, et suffisamment alimenté (TRAVADE et LARINIER, 1992).

Les expérimentations menées à Bedous et décrites dans la suite étaient destinées avant tout à évaluer l'efficacité de l'exutoire de dévalaison pour les smolts de saumon atlantique ; elles s'inscrivent aussi dans un programme à long terme visant à définir les critères de conception et les limites de fonctionnement des exutoires de surface au niveau des prises d'eau des centrales hydroélectriques et à mieux connaître les rythmes et les comportements de migration des espèces dévalantes (LARINIER et TRAVADE, 1997).

\section{MATÉRIEL ET MÉTHODES}

\section{Site d'étude}

L'aménagement de Bedous-Asasp est situé dans le sud-ouest de la France, sur le Gave d'Aspe, qui forme à sa confluence avec le Gave d'Ossau, le Gave d'Oloron (Figure 1). Le bassin versant au niveau de l'aménagement est de $366 \mathrm{~km}^{2}$; le module interannuel est de $16 \mathrm{~m}^{3} / \mathrm{s}$. Le régime des eaux est de type pluvio-nival : il se caractérise par de hautes eaux en hiver et au printemps et par de basses eaux de juillet à octobre. Les débits moyens mensuels lors de la période de dévalaison des smolts de saumon atlantique sont de $27 \mathrm{~m}^{3} / \mathrm{s}$ en mars, $35 \mathrm{~m}^{3} / \mathrm{s}$ en avril et $43 \mathrm{~m}^{3} / \mathrm{s}$ en mai.

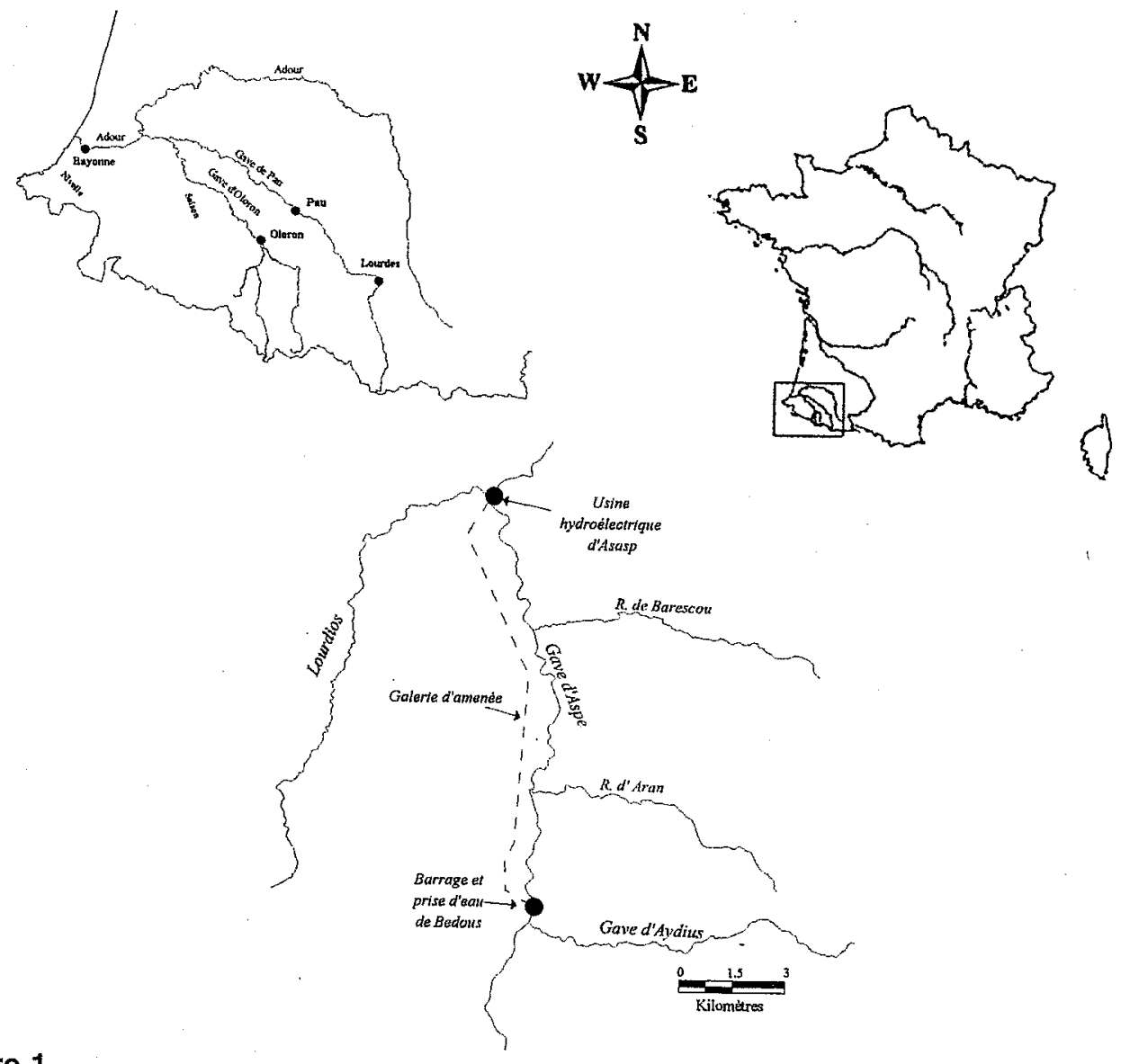

Figure 1

Situation géographique de l'aménagement de Bedous.

Figure 1

Geographical situation of the Bedous plant. 
L'ouvrage de Bedous (Figure 2 et Figure 3A) se compose d'un barrage mobile de $56 \mathrm{~m}$ de longueur équipé de deux vannes segments $(14 \mathrm{~m} \times 3 \mathrm{~m})$ à commande hydraulique et d'une vanne de chasse segment $(4 \mathrm{~m} \times 3,50 \mathrm{~m})$ en rive gauche surmontée d'un clapet. L'eau est dérivée dans un canal d'amenée de section trapézoïdale de $250 \mathrm{~m}$ de long et de largeur variant de 6 à 14 mètres. Une galerie de 12 kilomètres de longueur amène l'eau jusqu'à l'usine d'Asasp. L'usine est équipée de deux turbines Francis à axe vertical et turbine jusqu'à $25 \mathrm{~m}^{3} / \mathrm{s}$ sous $113 \mathrm{~m}$ de chute nominale pour une puissance fournie de 13,8 MW.
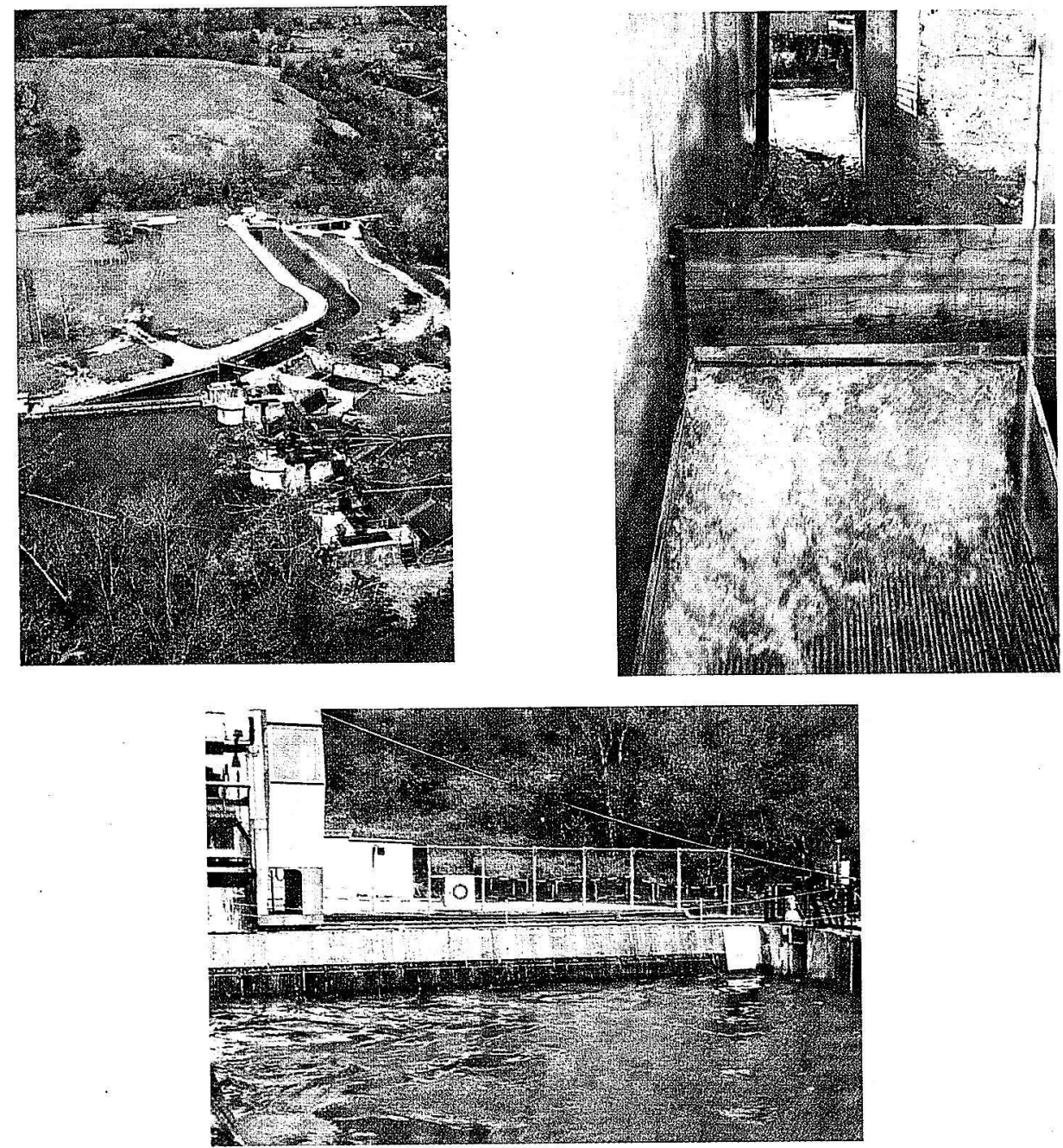

\section{Figure 2A}

Vue générale de la prise d'eau de Bedous.

\section{Figure 2B}

Vue amont des grilles de prise d'eau et de l'entrée de l'exutoire de dévalaison.

\section{Figure 2C}

Vue aval de l'exutoire de dévalaison.

\section{Figure 2A}

A general view of the Bedous water intake.

\section{Figure 2B}

An upstream view of the trashrack and of the bypass entrance.

\section{Figure 2C}

A downstream view of the bypass. 
La prise d'eau de $14 \mathrm{~m}$ de large est située à la jonction du canal d'amenée et de la galerie. Elle est protégée par une grille dont la section immergée est de $56 \mathrm{~m}^{2}$ et l'espacement entre barreaux de $30 \mathrm{~mm}$.

(A)
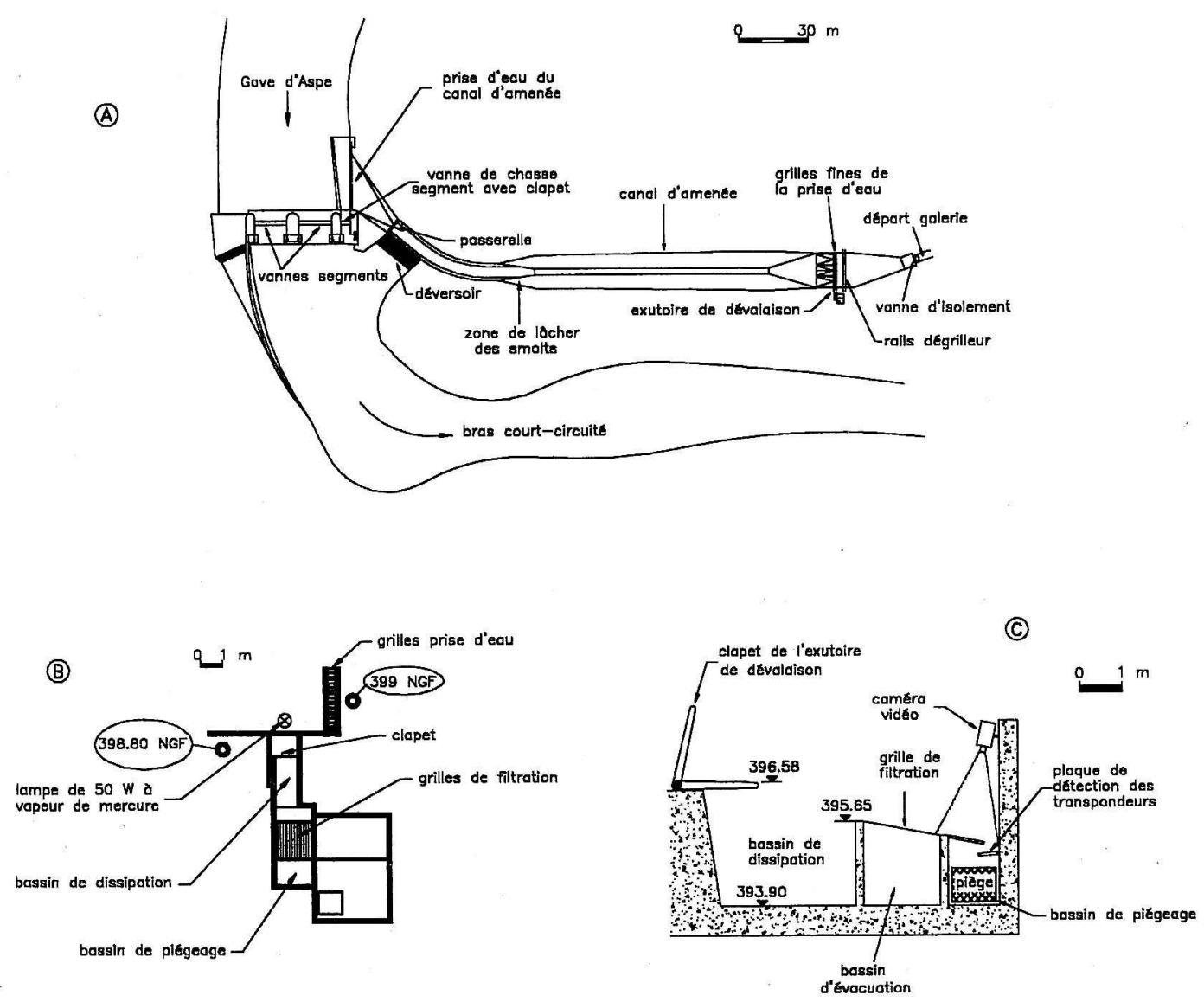

Figure 3A

Présentation générale de la prise d'eau de Bedous.

Figure 3B

L'exutoire de dévalaison.

Figure $3 \mathrm{C}$

Vidéo-surveillance des passages de poissons par l'exutoire et détection des smolts marqués par transpondeur.

Figure 3A

General presentation of the Bedous water intake.

Figure 3B

The downstream bypass.

Figure $3 \mathrm{C}$

Video monitoring of fish passages into the bypass and detection of the smolts marked with a transponder. 


\section{Dispositif de dévalaison}

Le dispositif de dévalaison (Figure 3B), construit en 1995, est constitué d'un exutoire de surface de $1 \mathrm{~m}$ de largeur situé latéralement en rive droite, à $1 \mathrm{~m}$ à l'amont du plan des grilles, puis d'un bassin de dissipation prolongé par une grille inclinée guidant les poissons vers un piège de contrôle (Figure $3 \mathrm{C}$ ). Le débit d'alimentation du dispositif est régulé par un clapet de $0,70 \mathrm{~m}$ de largeur et de $1,50 \mathrm{~m}$ de longueur asservi au niveau d'eau dans le canal d'amenée. Les débits dans l'exutoire ont été réglés entre $0,4 \mathrm{~m}^{3} / \mathrm{s}$ et $0,7 \mathrm{~m}^{3} / \mathrm{s}$ en 1995 (soit de $1,6 \%$ à $4,3 \%$ du débit dans le canal) et entre $0,5 \mathrm{~m}^{3} / \mathrm{s}$ et $1,2 \mathrm{~m}^{3} / \mathrm{s}$ en 1998 (soit de $2,8 \%$ à $4 \%$ du débit turbiné).

(A)

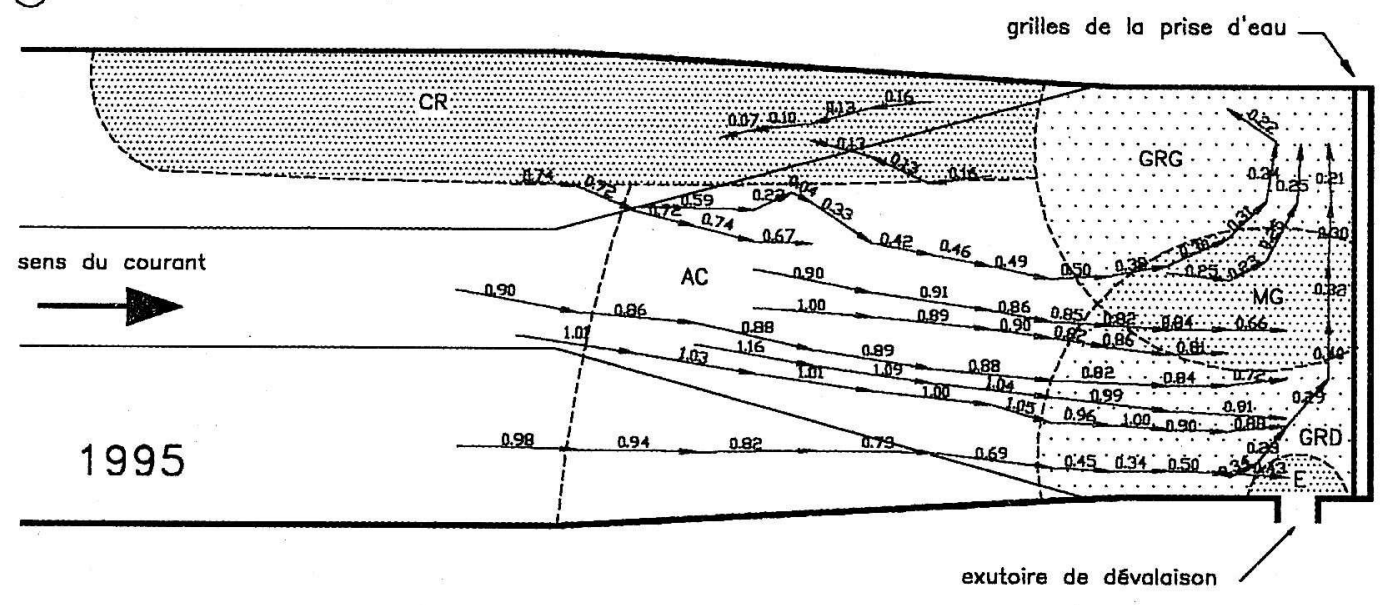

(B)

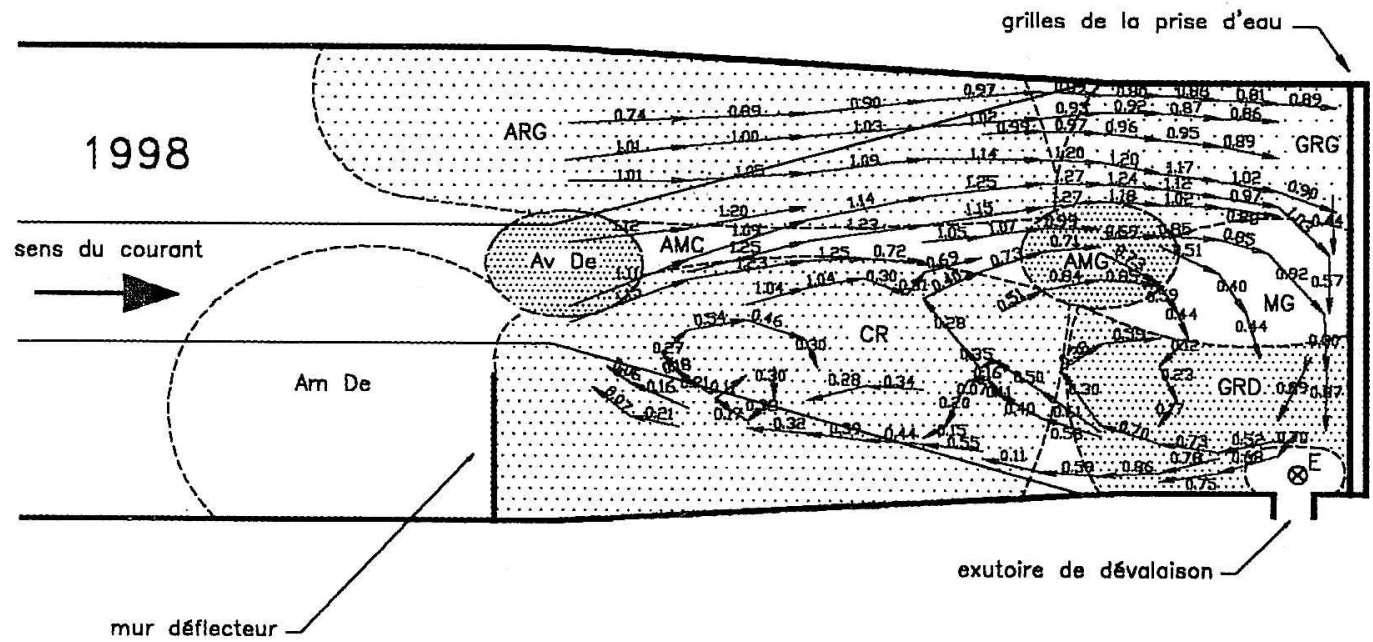

\section{Figure 4A}

Zones de réception des radio-émetteurs et hydrodynamique du canal d'amenée en 1995.

\section{Figure 4B}

Zones de réception des radio-émetteurs et hydrodynamique du canal d'amenée en 1998.

\section{Figure 4A}

Radiotelemetry listening areas and hydrodynamic in the intake canal in 1995.

\section{Figure 4B}

Radiotelemetry listening areas and hydrodynamic in the intake canal in 1998. 


\section{Hydrodynamique du canal d'amenée}

La présence d'une courbe dans la partie amont du canal d'amenée induit une dissymétrie très nette de l'écoulement, qui se traduit par une alimentation préférentielle de la prise d'eau en rive droite, un courant tangentiel au plan de grilles dirigé vers la rive gauche et une zone de recirculation d'environ $4 \mathrm{~m}$ de large en rive gauche qui s'étend jusqu'à une trentaine de mètres à l'amont des grilles (Figure 4A).

Suite à une première évaluation de l'efficacité de l'exutoire, efficacité jugée insuffisante, et à une étude sur modèle réduit physique des conditions hydrauliques dans le canal d'amenée, effectuée à l'Institut de Mécanique des Fluides de Toulouse (CHORDA et LARINIER, 1997), un mur déflecteur a été mis en place en 1997, en rive droite et à une trentaine de mètres des grilles de prise d'eau. L'objet de ce déflecteur était d'inverser l'écoulement dans le canal d'amenée, c'est-à-dire d'orienter l'écoulement principal vers la rive gauche : lors de l'expérimentation effectuée en 1998, l'alimentation préférentielle de la prise d'eau se situe désormais en rive gauche, le courant tangentiel au plan de grilles est dirigé vers la rive droite et la zone de recirculation d'environ $6 \mathrm{~m}$ de large se situe en rive droite et s'étend jusqu'au niveau du déflecteur (Figure 4B).

Des lâchers de flotteurs, l'utilisation de traceurs (fluorescéine) et des mesures de vitesses sur le site ont permis de caractériser les conditions hydrauliques dans le canal d'amenée. Des mesures effectuées sur le modèle réduit à l'Institut de Mécanique des Fluides de Toulouse ont permis de compléter les observations réalisées sur le terrain.

\section{Période d'étude}

Les expérimentations menées en 1995 et 1998 se sont déroulées des mois de mars à mai, ce qui englobe la période naturelle de migration de dévalaison des smolts de saumon atlantique dans la partie méridionale de l'Europe (BAGLINIERE, 1976 ; BOUSQUET, 1979 ; BOEUF, 1994).

\section{Paramètres du milieu}

Sept paramètres ont fait l'objet d'enregistrements pendant l'étude : la température de l'eau et de l'air, le rayonnement solaire global, la puissance fournie par les deux turbines de l'usine d'Asasp, la cote du clapet de la vanne de chasse du barrage et le niveau d'eau dans la retenue de façon à évaluer les débits déversés au barrage, le niveau dans le canal d'amenée et la cote du clapet de l'exutoire afin de déterminer le débit transitant par le dispositif de dévalaison.

Les données ont été recueillies sur des centrales d'acquisition portables autonomes de type Logicap (Centrales CR2M SAB 600 LUS-IP) conditionnées par Hydro-Invest.

Des mesures régulières de la transparence de l'eau ont également été réalisées à l'aide d'un disque de Secchi.

\section{Contrôle de l'efficacité de l'exutoire de dévalaison}

L'efficacité du dispositif de dévalaison a été évaluée à l'aide de la technique de marquage-recapture. La population dévalante issue d'une reproduction naturelle ou d'alevinage étant trop peu importante et difficile à piéger à l'amont du barrage de Bedous, les poissons utilisés ont été des smolts de saumon atlantique élevés en pisciculture.

Les poissons, mesurés et pesés, ont été marqués par ablation partielle d'une ou plusieurs nageoires (pectorale, pelvienne ou caudale), par projection intradermique d'un 
colorant (bleu alcyan) sur une de ces nageoires ou par la combinaison des deux types de marques.

Les smolts ont été lâchés par lots comprenant plusieurs dizaines d'individus (100 poissons par lot en moyenne en 1995 et 70 en 1998) de façon à créer un effet de banc, les poissons en dévalaison présentant généralement un comportement grégaire (THORPE et MORGAN, 1978 ; KYNARD, 1993) afin de lutter notamment contre la prédation (GINETZ et LARKIN, 1976 ; SMITH, 1985 ; PITCHER et PARRISH in PITCHER, 1993). Chaque lot de poissons a été identifié grâce à un marquage individualisé.

Les poissons ont été déversés dans le canal d'amenée de Bedous, à $120 \mathrm{~m}$ des grilles de prise d'eau et à raison de deux à trois lots par semaine sur la période d'étude. Les lâchers sont effectués de nuit, les smolts présentant en milieu naturel une activité de dévalaison généralement nocturne (SOLOMON, 1975 ; HOAR, 1976 ; THORPE, 1981 ; BOURGEOIS et O'CONNELL, 1988 ; MILLS, 1989 ; HANSEN et al., 1989 ; WOOD et al., 1993) même si en fin de période de migration, l'activité de dévalaison peut parfois devenir diurne (THORPE et MORGAN, 1978 ; LARINIER et BOYER-BERNARD, 1991a). Dans la partie méridionale de l'aire de répartition, les rythmes de dévalaison peuvent être cependant bimodaux, soir et matin (BAGLINIERE, 1976). De plus, au niveau des obstacles à la migration, ces rythmes semblent être également essentiellement nocturnes, les smolts étant majoritairement piégés de nuit (LARINIER et BOYER-BERNARD, 1991b ; TREMBLAY, 1993 ; TRAVADE et al., 1996 ; GOSSET et al., 1997 ; GOSSET et al., 1998).

La répétition des lâchers présente l'intérêt de pouvoir tester l'influence de facteurs abiotiques comme par exemple le débit, sur l'efficacité de l'exutoire de dévalaison. Dix-neuf lots d'une centaine de smolts ont ainsi été lâchés en 1995 et vingt lots d'environ 70 individus en 1998. L'efficacité du dispositif de dévalaison a été déterminée pour chaque lot par le pourcentage de smolts capturés dans le piège.

En 1998, vingt-cinq smolts par lot (excepté le premier lot), soit 475 poissons, ont été marqués à l'aide de transpondeurs ; ce sont des marques cylindriques de $11 \mathrm{~mm}$ de long et $2 \mathrm{~mm}$ de diamètre qui sont placées dans la cavité générale à l'aide d'une aiguille et qui possèdent un code alphanumérique permettant d'individualiser les individus. Ces marques n'excèdent pas $0,2 \%$ du poids des poissons marqués. Une plaque de détection installée à l'aval immédiat de la grille de filtration, juste avant le piège, a permis de connaître avec précision les heures de passage des poissons au niveau de l'exutoire (Figure $3 \mathrm{C}$ ). Ce marquage par transpondeur est une technique particulièrement performante compte tenu de son faible taux de perte et de son absence d'impact notamment sur la survie des juvéniles (OMBREDANE, BAGLINIERE et MARCHAND, 1998).

\section{Etude du comportement des smolts dans le canal d'amenée}

Si la technique de marquage-recapture permet l'évaluation de l'efficacité d'un exutoire de dévalaison, elle ne permet pas, par contre, d'en expliquer les causes liées avant tout au comportement du poisson.

La technique de radio-pistage a été utilisée lors des deux expérimentations afin de suivre les déplacements de smolts dans le canal d'amenée en amont des grilles et à l'approche de l'exutoire. Cette technique permet de mieux comprendre les stratégies d'exploration développées par les poissons à l'amont immédiat de la prise d'eau et d'appréhender l'influence de paramètres biotiques ou abiotiques sur leur comportement.

Un smolt porteur d'un radio-émetteur a été lâché au sein de chaque lot destiné aux opérations de marquage-recapture. 
Le suivi des smolts par radio-pistage a été effectué à l'aide de matériel d'origine américaine de marque ATS (Advanced Telemetry System).

Les émetteurs sont à fréquence individualisée dans la gamme des 48-49 Mhz. Les émetteurs utilisés ont une longueur moyenne de $16 \mathrm{~mm}$, un diamètre de $7 \mathrm{~mm}$ et un poids voisin de $1,3 \mathrm{~g}$ qui ne représente jamais plus de $3 \%$ du poids du poisson. Leur durée de vie est de 12 jours. L'émetteur est placé dans l'estomac des poissons à l'aide d'un tube pousseur. Cette technique de marquage paraît la plus appropriée à cette étude car la très grande majorité des smolts a dévalé dans les premières heures suivant le lâcher. D'après PEAKE et al. (1997), seul ce mode de marquage n'altère pas les capacités de nage des smolts de saumon atlantique.

Des postes fixes, composés d'un récepteur (modèle “ATS 2100 ") et d'un enregistreur numérique de 229 Ko de mémoire (modèle «D5040 Data Collection Computer "), sont reliés à des antennes réceptrices immergées. Ces postes permettent de surveiller en continu des zones de réception calibrées et de suivre ainsi précisément les déplacements des smolts radiomarqués. Afin de comparer le comportement des poissons lors des deux expérimentations, un certain nombre de zones de réception communes ont été individualisées (Figures 4A et 4B).

La récupération des données stockées par les enregistreurs numériques a été effectuée à l'aide d'un micro-ordinateur portable PC COMPAQ.

\section{Influence de la lumière}

De nombreuses études ont révélé que la lumière était susceptible, la nuit, de stopper les déplacements des poissons vers l'aval (HANSEN et JONSSON, 1985), de les attirer (FIELDS et al, 1958 ; KEMA, 1982 ; HAYMES, PATRICK et ONISTO, 1984 ; KAWAMURA, 1986 ; NEMETH et ANDERSON, 1992) ou de les guider vers des by-pass (TAFT, 1988 ; PUCKETT et ANDERSON, 1988 ; LARINIER et BOYER-BERNARD, 1991b ; EPRI, 1994). De plus, les poissons semblent rester à une certaine distance de la source lumineuse et préférer les zones de faible intensité lumineuse (HAYMES, PATRICK et ONISTO, 1984 ; KAWAMURA, 1986 ; TAFT, 1988 ; EPRI, 1994).

Une lampe à vapeur de mercure de faible puissance $(50 \mathrm{~W})$ a été installée au niveau de l'entrée de l'exutoire de dévalaison, à $1 \mathrm{~m}$ au-dessus de la surface de l'eau. Un programmateur a permis de régler l'alternance des conditions d'éclairage. En 1995, les différentes modifications apportées au cours de l'expérimentation ne permettent pas d'étudier les effets réels de cette lampe. En 1998, les conditions sont restées identiques durant toute l'étude : des cycles lumineux de $15 \mathrm{~min}$, composés d'une période d'éclairage de 10 min suivie d'une période d'obscurité de $5 \mathrm{~min}$, se sont succédés toutes les nuits.

Une surveillance vidéo a été assurée en continu tout au long de l'expérimentation : une caméra vidéo (Panasonic WV-7140E) associée à deux magnétoscopes à temps échelonné (Panasonic AG-6730) a été installée au-dessus de la grille de filtration de l'exutoire pour enregistrer les passages nocturnes de poissons (Figure $3 \mathrm{C}$ ).

\section{RÉSULTATS DE L'ÉTUDE MENÉE EN 1995}

\section{Efficacité de l'exutoire de dévalaison}

Dix-neuf lots d'une centaine de smolts chacun ont été lâchés dans le canal d'amenée, soit au total 1899 poissons. Trois cent vingt-six poissons ont été récupérés dans le piège, ce qui correspond à une efficacité moyenne de l'exutoire de dévalaison de $17,1 \%$. Selon les lots, les pourcentages de recapture ont varié de $9,2 \%$ à $30,6 \%$. 


\section{Activité de dévalaison}

Soixante-huit pour cent des poissons recapturés (222 sur 326) l'ont été la nuit de leur lâcher, la majorité d'entre eux (200 poissons soit $90 \%$ ) empruntant l'exutoire dans l'heure suivant leur remise à l'eau. Sur les cent quatre smolts qui sont passés les jours suivants, $90 \%$ ont été piégés de nuit.

\section{Effet de la taille des smolts}

Huit cent trois smolts lâchés et 150 récupérés dans le piège ont été mesurés lors de l'étude. La taille moyenne des smolts recapturés $(19,1 \mathrm{~cm})$ est légèrement supérieure à celle des poissons lâchés $(18,6 \mathrm{~cm})$, la différence étant statistiquement significative (ANOVA, $F=14,36 ; p<0,0005$ ).

La Figure 5 présente les fréquences par classes de taille des poissons lâchés et des smolts piégés. L'analyse des données révèle que le pourcentage des poissons appartenant aux classes de taille $15-18 \mathrm{~cm}$ est plus faible sur les poissons récupérés que sur les poissons lâchés (WILCOXON, $p<0,01$ ), alors que c'est l'inverse sur les poissons appartenant aux classes de taille 20-22 cm (WILCOXON, $p<0,05$ ).

1995

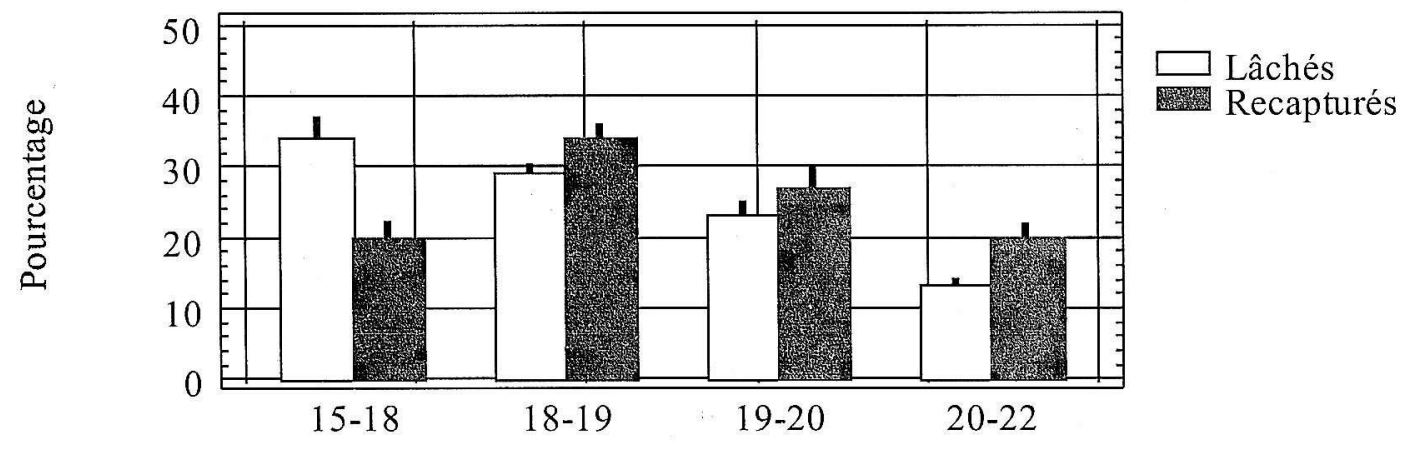

\section{Figure 5}

Répartition des tailles (Lt en $\mathrm{cm}$ ) des smolts lâchés et des smolts recapturés en 1995.

\section{Figure 5}

Lengths repartition ( $\mathrm{Lt}$ in $\mathrm{cm}$ ) of smolts released and captured in 1995.

\section{Comportement des poissons radiomarqués}

Dix-neuf smolts radiomarqués ont été lâchés au cours de l'étude et sept ont été capturés dans le piège, soit $36,8 \%$. Ce taux de recapture est nettement supérieur à celui obtenu à l'aide de la technique de marquage-recapture. Le faible nombre de poissons équipés d'un émetteur ne permet pas toutefois de tirer de réels enseignements de ces résultats.

Les déplacements du dernier smolt lâché n'ont pu être suivis en raison de problèmes de fonctionnement des enregistreurs. 
Les délais entre le lâcher et l'arrivée au niveau des grilles sont très variables : ils sont compris entre $1 \mathrm{~min}$ et plus de $15 \mathrm{~h}$.

Quatorze poissons, soit $77,7 \%$, se sont présentés la première fois au niveau des grilles de prise d'eau en rive droite, rive sur laquelle se situe l'écoulement principal.

Les durées de présence des poissons dans le canal d'amenée une fois qu'ils ont été reçus à l'amont immédiat des grilles varient de 1 min à près de $85 \mathrm{~h}, 16$ smolts y

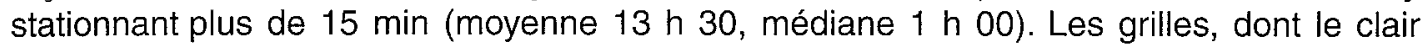
entre barreaux est de $30 \mathrm{~mm}$, semblent exercer un effet répulsif sur les poissons.

Les zones de réception individualisées dans le canal d'amenée ne sont pas toutes fréquentées avec la même intensité (ANOVA, $F=7,79 ; p<0,0001$ ). Les smolts stationnent préférentiellement en rive gauche (Figure 6) : ils demeurent $38,9 \%$ et $25,2 \%$ de leur temps de présence dans le canal d'amenée respectivement dans la zone rive gauche (GRG) et dans le courant de recirculation (CR). La rive droite (GRD) et donc l'entrée de l'exutoire de dévalaison (E) sont peu fréquentées et la zone correspondant au milieu du canal à l'amont immédiat des grilles (MG), soumise au courant tangentiel au plan de grilles, n'est qu'une zone de passage. Les poissons séjournent $22,5 \%$ de leur temps dans la zone englobant le milieu du canal et la rive droite à l'amont de la prise d'eau (AC). Quand les poissons sont reçus dans cette zone, ils stationnent principalement en rive gauche, la zone CR ne couvrant pas réellement toute la largeur du courant de recirculation.

Treize smolts sur dix-huit, soit $72,2 \%$, ont été reçus au moins une fois au niveau de l'entrée du dispositif de dévalaison. Les durées de stationnement pour ces poissons ont varié de $5 \mathrm{~s}$ à plus de $7 \mathrm{~min}$. Six smolts se sont présentés devant l'entrée de l'exutoire mais ne se sont pas engagés à l'intérieur. De plus, quatre des sept individus radiomarqués recapturés n'ont pas pénétré dans le by-pass lors de leur première venue au niveau de l'entrée. L'attractivité de l'exutoire ne semble pas optimale, l'écoulement principal en rive droite limitant fortement la zone d'influence du dispositif.

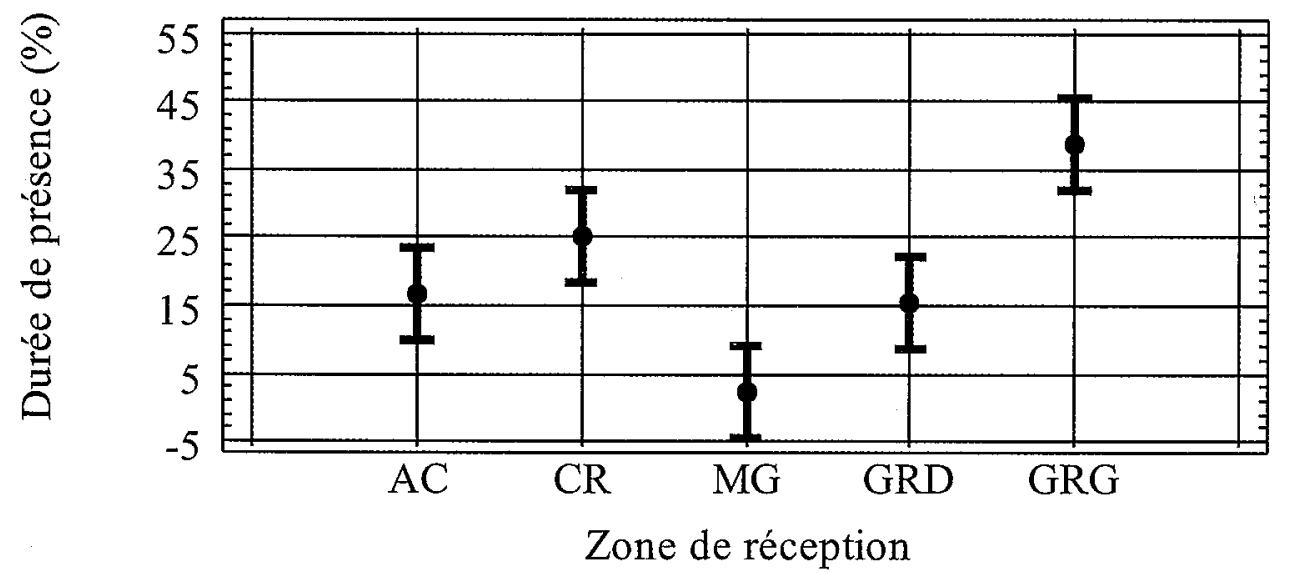

\section{Figure 6}

Pourcentages de temps de présence des smolts radiomarqués dans les
différentes zones de réception en 1995.

\section{Figure 6}

Presence time percentages of radiotagged smolts in the different listening areas in 1995. 


\section{Influence du débit dans le canal d'amenée sur l'efficacité de l'exutoire}

Le débit transitant dans le canal d'amenée semble avoir un effet sur l'efficacité de l'exutoire de dévalaison : $7,2 \%$ des smolts ont été piégés les nuits de lâcher pour des débits supérieurs à $20 \mathrm{~m}^{3} / \mathrm{s}$ contre $16,4 \%$ pour des débits inférieurs, la différence étant statistiquement significative (ANOVA, $F=6,31 ; p<0,05$ ).

Le débit dans le canal d'amenée par contre ne semble pas avoir d'influence sur les durées de stationnement des poissons radiomarqués dans les différentes zones de réception individualisées (ANOVA, $F=0,03 ; p>0,05$ ).

\section{RÉSULTATS DE L'ÉTUDE MENÉE EN 1998}

\section{Efficacité de l'exutoire de dévalaison}

Vingt lots d'environ 70 poissons ont été lâchés, soit 1422 individus. Sept cent quatre-vingt-douze smolts ont été piégés, ce qui correspond à une efficacité moyenne de l'exutoire de $55,5 \%$. L'efficacité varie suivant les lots de $38,4 \%$ à $74,3 \%$.

\section{Activité de dévalaison}

Sur 792 poissons, 659 individus, soit $83 \%$ des smolts piégés dans l'exutoire de dévalaison, ont été recapturés dans la première heure suivant le lâcher. Sur les 250 poissons marqués par transpondeur, 196 , soit $78,4 \%$, ont également été piégés dans l'heure suivant le lâcher, le délai moyen de passage dans l'exutoire la nuit de lâcher étant de $18 \mathrm{~min}$.

Deux cent douze smolts ou pré-smolts de saumon atlantique ne provenant pas des opérations de marquage-recapture ont été capturés dans le piège. Ces poissons peuvent provenir d'une reproduction naturelle en amont de l'aménagement mais aussi d'un repeuplement effectué par le Conseil Supérieur de la Pêche au printemps 1997. Cent quatre-vingt-treize poissons ont été piégés de nuit, ce qui correspond à $91 \%$ des individus. Deux pics de migration correspondant à $78,3 \%$ des poissons ont pu être individualisés : le premier du 20 au 26 avril (104 smolts) et le second du 7 au 12 mai (62 smolts). Ils ont été tous les deux précédés d'une augmentation de plus de $2{ }^{\circ} \mathrm{C}$ en une semaine des températures moyennes journalières de l'eau, les débits demeurant relativement stables.

1998

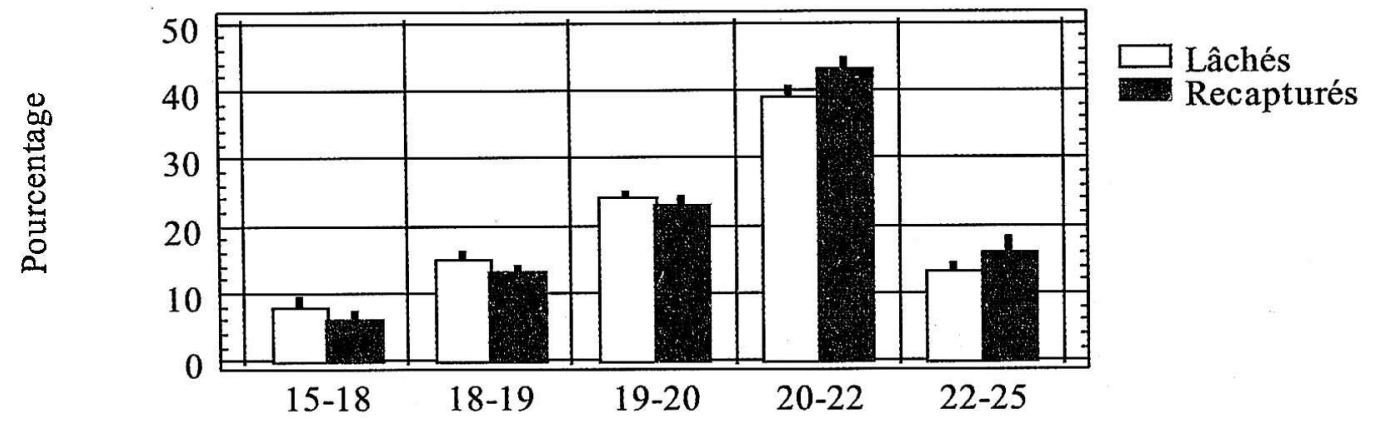

Figure 7

Répartition des tailles ( $\mathrm{Lt}$ en $\mathrm{cm}$ ) des smolts lâchés et des smolts recapturés en 1998.

\section{Figure 7}

Lengths repartition ( $\mathrm{Lt}$ in $\mathrm{cm}$ ) of smolts released and captured in 1998. 


\section{Effet de la taille des smolts}

La longueur totale moyenne des poissons lâchés est de $20,2 \mathrm{~cm}$ et celle des smolts piégés de $20,5 \mathrm{~cm}$, la différence étant statistiquement significative (ANOVA, $F=11,29$; $p<0,001)$. La Figure 7 présente les fréquences par classes de taille des smolts lâchés et des individus recapturés dans le piège. Lanalyse des données révèle que les pourcentages des poissons appartenant aux classes de taille $15-18 \mathrm{~cm}$ et $18-19 \mathrm{~cm}$ sont plus faibles sur les poissons récupérés que sur les poissons lâchés (WILCOXON, $p<0,05$ et $p<0,01$ ), alors que c'est l'inverse sur les poissons appartenant à la classe de taille 20-22 cm (WILCOXON, $p<0,05$ ).

De même, les smolts radiomarqués récupérés dans le piège sont de taille supérieure à ceux qui passent à travers les grilles de prise d'eau (WMW, $p<0,005)$.

\section{Comportement des poissons radiomarqués}

Neuf des vingt smolts radiomarqués, soit $45 \%$, ont été récupérés dans le piège. Ce pourcentage est comparable à celui obtenu avec la technique de marquage-recapture $(55 \%)$.

Deux émetteurs se sont éteints dans les premières minutes suivant le lâcher. Seuls les déplacements de dix-huit poissons ont donc été suivis dans le canal d'amenée.

A l'exception de deux poissons qui sont restés respectivement $1 \mathrm{~h} 43$ et $5 \mathrm{~h} 51$ à proximité du point de lâcher, les temps mis par les autres smolts pour dévaler les $120 \mathrm{~m}$ séparant le point de lâcher des grilles de prise d'eau sont peu importants. Ils varient de $1 \min 29$ à 6 min 55, le temps moyen étant de 3 min.

Quinze poissons, soit $83,3 \%$, arrivent au niveau des grilles par la rive gauche, rive sur laquelle se trouve l'écoulement principal.
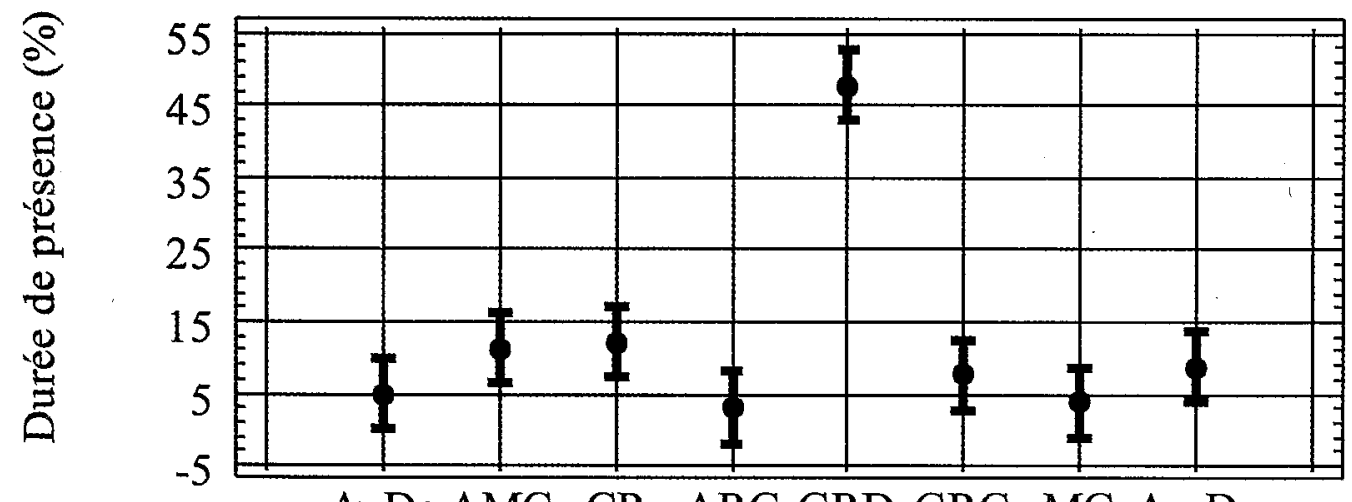

AvDe AMC CR ARG GRD GRG MG AmDe

Zone de réception

Figure 8

Pourcentages de temps de présence des smolts radiomarqués dans les différentes zones de réception en 1998.

\section{Figure 8}

Presence time percentages of radiotagged smolts in the different listening areas in 1998. 
Quatre smolts sont restés moins de deux minutes dans le canal d'amenée une fois arrivés devant les grilles, deux poissons sont passés à travers les grilles de prise d'eau et deux ont emprunté l'exutoire de dévalaison. En ce qui concerne les autres individus, les temps sont variables et sont compris entre 2 min 22 et plus de $23 \mathrm{~h}$ (moyenne $2 \mathrm{~h} 15$ min, médiane $8 \min 50)$.

Les différentes zones de réception individualisées dans le canal d'amenée ne sont pas fréquentées avec la même intensité par les smolts (ANOVA, $F=17,63 ; p<0,0001$ ). Les zones les plus fréquentées par l'ensemble des smolts sont celles situées sur la rive droite du canal d'amenée, dans la zone de recirculation située à l'opposé de l'écoulement principal (Figure 8). Les poissons passent plus de $60 \%$ de leur temps de présence dans le canal sur cette rive dont $47,8 \%$ dans la zone GRD qui inclut l'entrée du dispositif de dévalaison. Les poissons stationnent par contre peu de temps en rive gauche : $7,7 \%$ dans la zone GRG et $3 \%$ en ARG. La zone MG soumise aux courants tangentiels au plan de grilles est également peu fréquentée et ne constitue qu'une zone de passage. La partie de canal située à l'amont et à l'aval du mur déflecteur (zones AvDe et AmDe) est faiblement fréquentée par les poissons.

Seize des dix-huit smolts (soit $88,8 \%$ ) pour lesquels des données complètes ont été recueillies se sont présentés au moins une fois au niveau de l'entrée de l'exutoire. Les deux poissons qui n'ont pas été reçus dans cette zone ont traversé les grilles en moins d'une minute. Neuf seulement de ces seize poissons ont emprunté le dispositif : trois smolts se sont engagés à l'intérieur lors de leur première incursion au niveau de l'entrée de l'exutoire, trois au bout de leur deuxième incursion et trois enfin après s'y être présentés entre 3 et 5 fois. Les durées de stationnement varient de 8 secondes à plus de 50 minutes, 8 poissons ayant été reçus plus de $1 \mathrm{~min} 30$. L'attractivité de l'exutoire ne paraît pas optimale. Le courant transversal marqué au niveau des grilles, dirigé de la rive gauche vers la rive droite, induit de fortes ascendances au niveau du dispositif de dévalaison, qui tendent à réduire sa zone d'influence et à masquer son entrée.

\section{Influence du débit dans le canal d'amenée}

Dans les analyses qui suivent, deux classes de débit dans le canal d'amenée ont été distinguées, l'une inférieure à $20 \mathrm{~m}^{3} / \mathrm{s}$, l'autre supérieure à $24 \mathrm{~m}^{3} / \mathrm{s}$.

L'efficacité de l'exutoire, les nuits de lâcher, dépend du débit dans le canal d'amenée (WMW, $p<0,05)$. Les pourcentages de recapture sont en effet plus élevés lorsque les débits turbinés sont supérieurs à $24 \mathrm{~m}^{3} / \mathrm{s}$.

Le débit a également un effet sur les pourcentages de présence des poissons radiomarqués dans les différentes zones de réception individualisées dans le canal d'amenée (ANOVA, $F=4,74 ; p<0,0001$ ). Pour des débits turbinés supérieurs à $24 \mathrm{~m}^{3} / \mathrm{s}$, les pourcentages de présence des smolts dans les zones situées en rive droite augmentent, notamment la zone GRD qui devient près de deux fois plus fréquentée. Les poissons stationnent nettement moins de temps dans les zones situées en rive gauche (GRG et $A R G$ ) ainsi que dans la zone AmDe à l'amont immédiat du mur déflecteur (Figure 9).

Par contre, aucune influence du débit dans l'exutoire ou du rapport débit dans l'exutoire sur débit dans le canal, n'a pu être mise en évidence sur l'efficacité du dispositif de dévalaison.

\section{Influence de l'éclairage de l'exutoire par une lampe à vapeur de mercure}

Le fonctionnement de la lampe à vapeur de mercure n'a aucun effet sur le stationnement des poissons dans les différentes zones de réception (ANOVA, $F=0,17$; $p>0,05$ ), le facteur hydraulique restant prépondérant. De plus, la zone la plus explorée 
par les poissons, à l'amont immédiat des grilles et en rive droite, englobe la zone soumise aux variations d'éclairage ce qui a tendance à masquer les effets potentiels de la lumière. L'activité des smolts dans les zones proches de la source lumineuse (zones 2, 3, 4, 6 et 7), mesurée par le nombre de changements de zones de réception par minute, est cependant influencée par le fonctionnement de la lampe. Les poissons soumis aux deux conditions d'éclairage réalisent en moyenne 1,9 incursion par minute dans l'une des zones précédemment citées durant l'obscurité contre 1,4 incursion lorsque la lampe est allumée, la différence étant statistiquement significative (WILCOXON, $p<0,05$ ). La lumière aurait tendance à fixer les poissons à proximité de la zone éclairée.
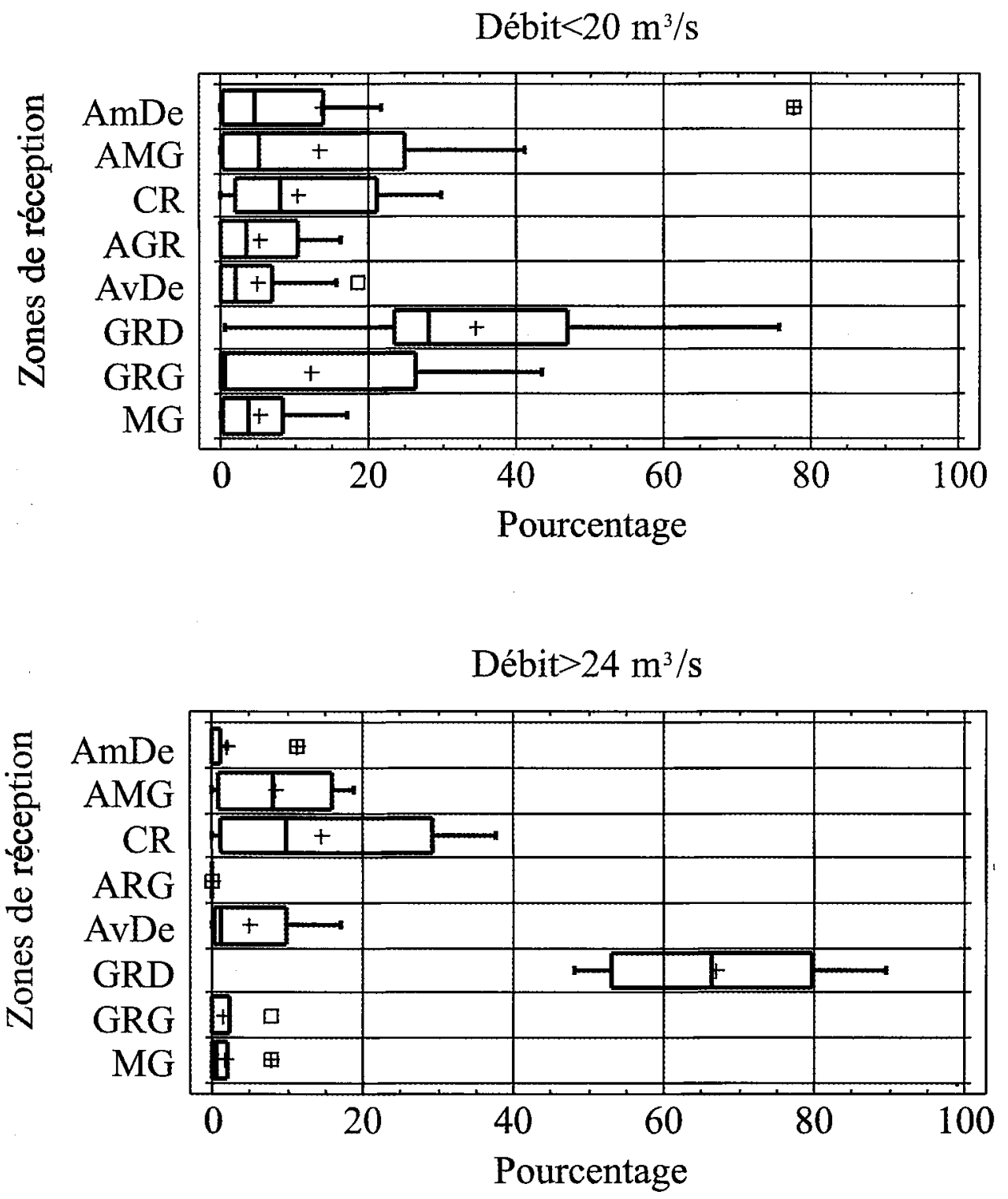

Figure 9

Pourcentages de temps de présence des smolts radiomarqués dans les différentes zones de réception en fonction du débit turbiné.

Figure 9

Presence time percentages of radiotagged smolts in the different listening areas according to the turbine discharge. 
Le fonctionnement de la lampe à vapeur de mercure située au-dessus de l'entrée de l'exutoire de dévalaison exerce un effet très net sur le passage des poissons par le dispositif de dévalaison. Lors de la première heure suivant les lâchers des smolts, il passe en moyenne 0,65 poisson par minute lorsque la lampe ne fonctionne pas et 0,32 lorsqu'elle est allumée (WMW, $\mathrm{p}<0,01)$. Cette période relativement courte présente l'intérêt d'inclure le piégeage d'une forte proportion des poissons marqués (659 smolts, soit $83,2 \%)$ et de concerner avant tout les smolts de saumon atlantique $(92,6 \%$ des individus piégés). En effet, si la surveillance vidéo permet de connaître les heures exactes de passage des poissons et donc les conditions d'éclairage lors des passages, elle ne permet pas par contre de distinguer les smolts de saumon des truites fario dévalantes.

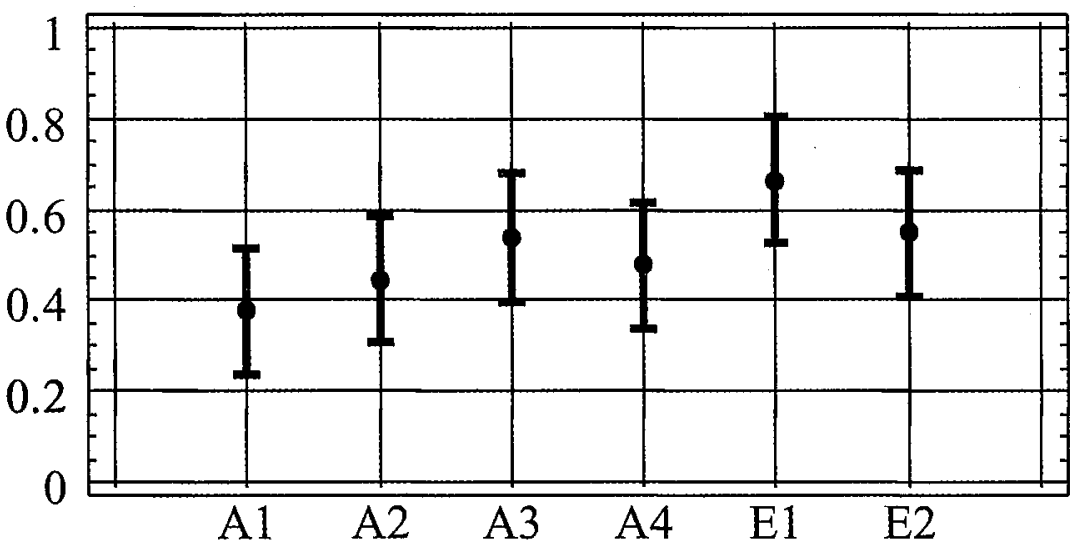

Code lumineux
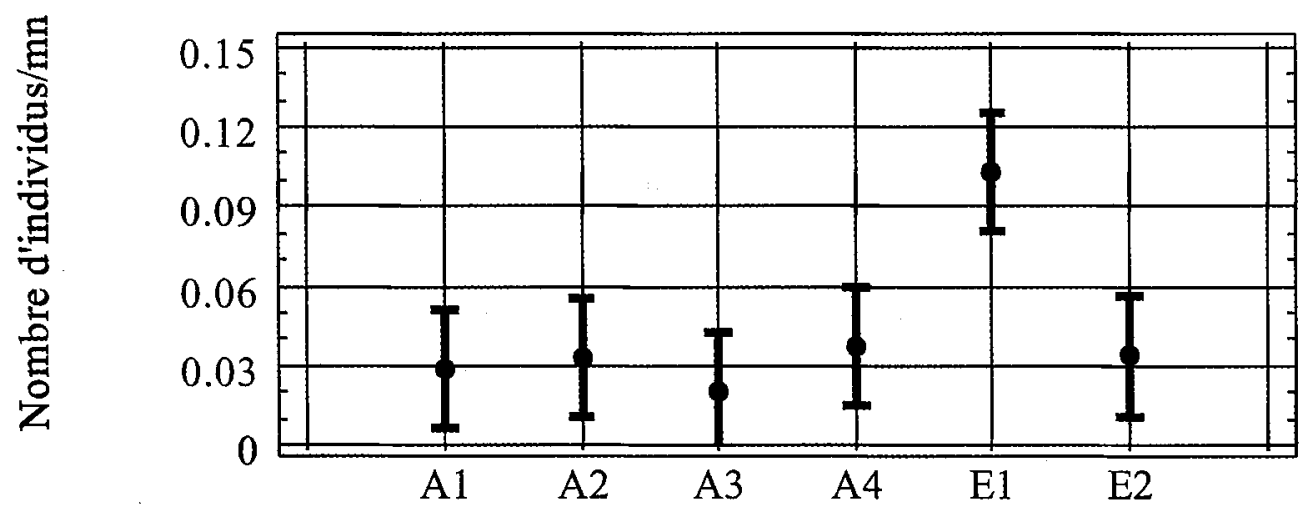

Code lumineux

Figure 10A

Nombre de smolts piégés durant la première heure suivant le lâcher en fonction des conditions lumineuses.

Figure 10B

Nombre de smolts marqués par transpondeur piégés les nuits de lâcher en fonction des conditions lumineuses.

Figure 10A

Number of smolts captured during the first hour following the release according to light conditions.

Figure 10B

Number of smolts marked with a transponder captured during the release night according to light conditions. 
Le passage des smolts équipés d'un transpondeur, dont les heures de passage sont connues avec précision, sont plus nombreux lorsque la lampe ne fonctionne pas $\left(x^{2}=7,78 ; p<0,005\right)$ : il passe en moyenne 1,47 poisson par heure contre 0,94 poisson par heure lorsque la lampe est allumée.

La comparaison des passages au niveau de l'exutoire les nuits durant lesquelles au minimum $77 \%$ des poissons capturés sont des truites, ce qui correspond à 211 individus, ne met en évidence aucune différence des passages par l'exutoire en fonction des conditions lumineuses (WMW, $p>0,1$ ).

Les cycles lumineux de 15 minutes ont été divisés en 6 périodes de 2 min 30 ( $A 1$, A2, A3 et A4 pour la période de fonctionnement de la lampe et E1 et E2 pour la période d'extinction). Lors de la première heure suivant le lâcher des smolts, il apparaît une différence significative des passages de poissons en fonction des conditions lumineuses (KRUSKAL-WALLIS, $p<0,05$ ). Les recaptures sont plus nombreuses lorsque la lampe ne fonctionne pas, principalement lors de la condition E1 qui correspond à la période suivant immédiatement l'extinction de la lampe. (Figure 10A).

Les passages des smolts munis de transpondeur les nuits de lâcher mettent également en évidence une augmentation des passages dans les premières minutes qui suivent l'extinction de la lampe (KRUSKAL-WALLIS, $p<0,05$ ). II passe en moyenne 0,031 poisson par minute en condition $E 1$ alors que les rythmes de passage restent inférieurs à 0,02 poisson par minute pour toutes les autres conditions lumineuses (Figure 10B).

\section{poissons}

Influence de l'hydrodynamique du canal d'amenée sur le comportement des

La mise en place d'un mur déflecteur en 1997 a eu pour effet de modifier considérablement les conditions hydrauliques à l'approche des grilles de la prise d'eau :

- l'écoulement principal à l'approche des grilles a été dévié de la rive droite vers la rive gauche ; droite ;

- la zone de recirculation, située à l'origine en rive gauche, a été déplacée en rive

- le sens des courants tangentiels au plan de grilles de prise d'eau a été inversé.

Le pincement de l'écoulement au niveau du mur déflecteur a eu pour conséquence d'augmenter les vitesses tangentielles au plan de grilles ainsi que la superficie de la zone de recirculation et les vitesses de courant dans cette zone.

La comparaison des résultats obtenus en 1995 et en 1998 peut permettre d'appréhender l'influence et l'importance des facteurs hydrauliques à l'approche de la prise d'eau sur le comportement de poissons en dévalaison.

En 1995, 14 poissons sur 18 (soit $77,7 \%$ ) arrivent au niveau des grilles de prise d'eau par la rive gauche alors qu'en 1998, 15 smolts sur 18 (soit $83,3 \%$ ) se présentent la première fois au niveau des grilles en rive droite.

La comparaison des pourcentages de présence des poissons dans les zones de réception communes aux deux expérimentations (Figure 11) met en évidence des différences considérables (ANOVA, $F=8,43 ; p<0,0001$ ). En 1995, les smolts passent plus de $66 \%$ de leur temps en rive gauche, dans les zones GRG $(34,4 \%)$ et CR $(31,9 \%)$. 
L'amont immédiat des grilles en rive droite (zone GRD) est peu fréquenté (16\%) et la zone exutoire très peu explorée (moins de $1 \%$ ). La zone MG correspond à une zone de passage où les poissons ne stationnent jamais longtemps. En 1998, les poissons stationnent près de $55 \%$ en rive droite à l'amont immédiat des grilles dont 16,6\% devant l'entrée de l'exutoire. La rive gauche du canal est peu explorée, les smolts n'y demeurant que $20,3 \%$ du temps. La zone MG, tout comme en 1995, correspond à une zone de passage.

\section{5}

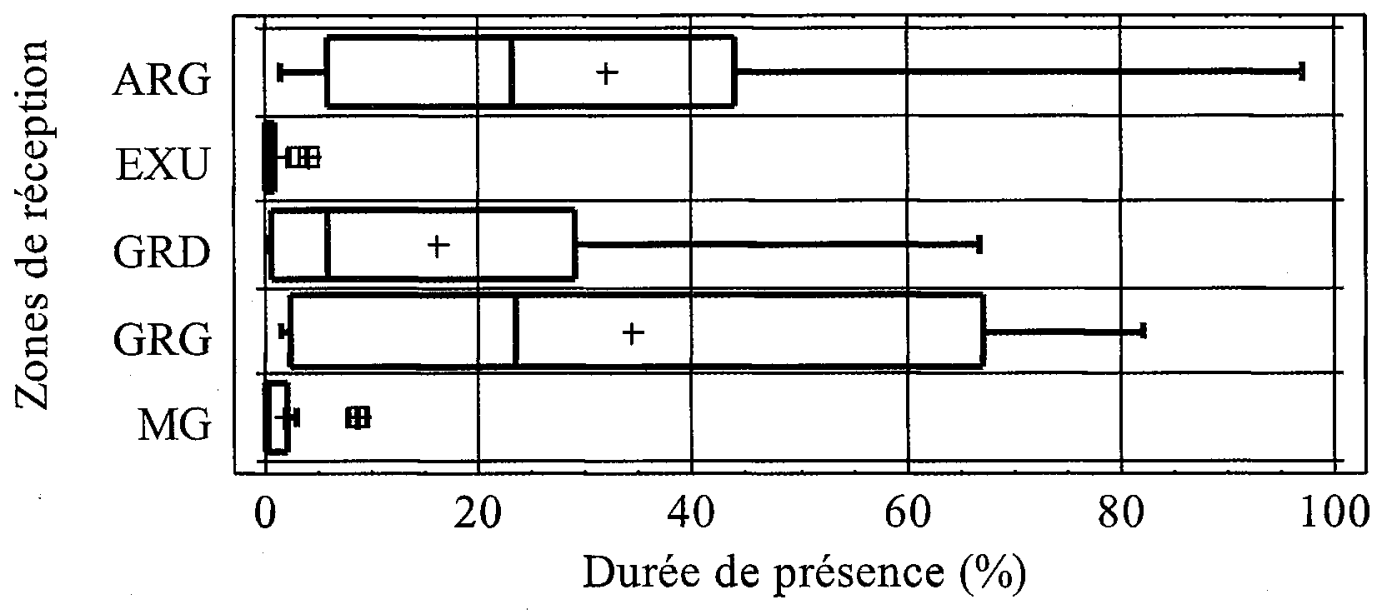

1998

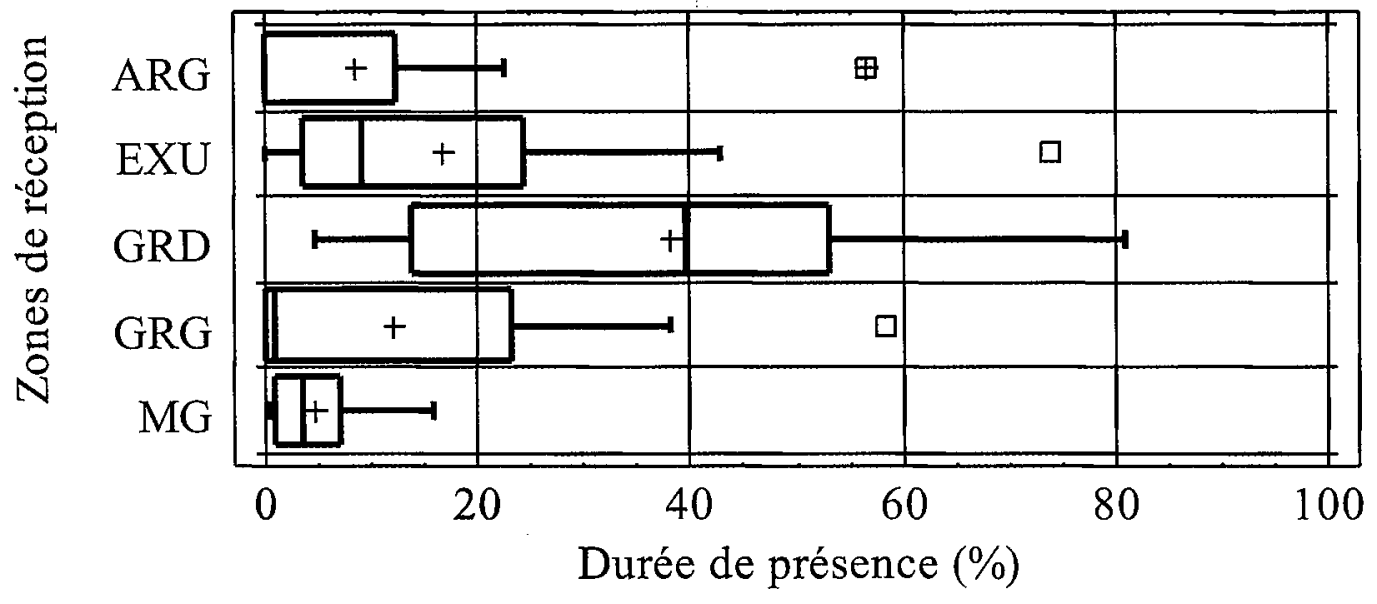

\section{Figure 11}

Comparaison des pourcentages de temps de présence des smolts radiomarqués dans les différentes zones de réception en 1995 et 1998.

\section{Figure 11}

Comparison of presence time percentages of radiotagged smolts in the different listening areas in 1995 and 1998. 
Le stationnement des poissons dans le canal d'amenée semble sous l'étroite dépendance du facteur hydrodynamique. Ainsi, lors des deux expérimentations, les poissons stationnent préférentiellement dans les zones correspondant au courant de recirculation qui se situe en rive gauche en 1995 et en rive droite en 1998. Les durées de stationnement plus importantes en rive droite en 1998, notamment aux abords de l'exutoire, expliquent très certainement la nette augmentation de l'efficacité du dispositif de dévalaison.

\section{DISCUSSION}

Une première évaluation de l'efficacité de l'exutoire installé à la prise d'eau de Bedous a été effectuée au printemps 1995 ; les opérations de marquage-recapture ont montré que celle-ci était notablement insuffisante : elle a varié de $9,2 \%$ à $30,6 \%$ suivant les lots pour une moyenne de $17 \%$. Le suivi des déplacements de smolts à l'aide de la technique de radio-pistage, à l'amont immédiat des grilles de prise d'eau, a permis de mettre en évidence l'influence des conditions hydrauliques dans le canal d'amenée sur le comportement des poissons. Les smolts stationnent préférentiellement dans la zone de recirculation, qui se trouve sur la rive opposée à l'écoulement principal et à l'exutoire de dévalaison.

Suite à cette première étude, un mur déflecteur, visant à modifier les conditions hydrauliques dans le canal, a été installé en rive gauche à une trentaine de mètres à l'amont des grilles. Il a entraîné une nette amélioration de l'efficacité du dispositif de dévalaison, qui est passée en 1998 à plus de $55 \%$ en moyenne et a varié suivant les lots de $38,4 \%$ à $74,3 \%$.

Une inversion des zones les plus fréquentées par les smolts a été constatée : en 1995, les poissons stationnent principalement en rive gauche, à l'opposé de l'exutoire de dévalaison, alors que la rive droite devient la plus fréquentée en 1998. Les durées de présence à proximité de l'entrée de l'exutoire sont nettement plus importantes en 1998. et expliquent très certainement l'efficacité largement supérieure du dispositif de dévalaison.

En 1998, les poissons stationnent d'autant plus sur la rive droite, notamment dans le courant de recirculation, que les débits dans le canal d'amenée sont importants. $\mathrm{Ce}$ comportement s'explique par le renforcement, lorsque le débit augmente, du courant tangentiel aux grilles qui améliore le guidage du poisson vers la rive droite.

Aucun effet du débit transitant dans l'exutoire sur l'efficacité de ce dernier n'a pu être mis en évidence, ni en 1995, ni en 1998. L'efficacité du dispositif de dévalaison semble avant tout liée à l'hydrodynamique générale du canal d'amenée à l'approche des grilles.

Les effets du débit transitant dans le canal d'amenée sur l'efficacité de l'exutoire sont opposés entre les deux expérimentations. En 1995, l'efficacité du dispositif diminue avec le débit alors qu'elle augmente en 1998. Lors de la première expérimentation, l'écoulement principal se situe en rive droite, à proximité de l'exutoire. L'augmentation du débit dans le canal d'amenée se traduit par une augmentation des vitesses qui ont tendance à masquer l'entrée du dispositif de dévalaison en réduisant sa zone d'influence et par conséquent son efficacité. De plus, les vitesses tangentielles au plan de grilles, qui ont tendance à guider les poissons vers la rive opposée à celle de l'exutoire, sont d'autant plus marquées que les débits sont importants (CHORDA et LARINIER, 1997). En 1998, les vitesses tangentielles augmentent également avec les débits mais elles guident les smolts vers la rive droite et donc vers l'entrée de l'exutoire. 
Le fonctionnement d'une lampe à vapeur de mercure placée au-dessus de la prise d'eau de l'exutoire en 1998 n'a pas paru modifier notablement les déplacements des smolts dans le canal, le facteur hydraulique restant prépondérant. Cependant, l'éclairage intermittent de l'entrée de l'exutoire modifie les rythmes de passage : les poissons sont plus nombreux à s'engager à l'intérieur du dispositif dans les premières minutes qui suivent l'extinction de la lampe. Ces résultats sont comparables à ceux obtenus par LARINIER et BOYER-BERNARD (1991b), LARINIER et TRAVADE (1997) ou CROZE, CHANSEAU et LARINIER (1999).

Un effet de la taille des smolts a pu être mis en évidence dans les deux études. L'efficacité de l'exutoire semble significativement plus importante pour les poissons appartenant aux classes de taille $20-22 \mathrm{~cm}$ que pour les poissons appartenant aux classes de taille inférieures à $18-19 \mathrm{~cm}$. Les grilles de prise d'eau dont le clair entre barreaux est de $30 \mathrm{~mm}$ exerceraient un effet répulsif moins marqué sur les individus de petite taille.

\section{CONCLUSION}

L'intérêt de cette expérimentation a été de mettre en évidence que le comportement des poissons à l'amont des grilles d'une prise d'eau était fortement dépendant des conditions hydrauliques : les smolts stationnent préférentiellement dans les zones de recirculation et de faible courant, évitant les vitesses les plus importantes. A partir de ces constatations, on a montré qu'il était possible d'améliorer significativement l'efficacité d'un exutoire de dévalaison en modifiant de façon relativement simple les conditions hydrauliques dans le canal d'amenée. Ces résultats ouvrent des perspectives intéressantes pour régler de façon élégante le problème de la dévalaison au niveau de certaines microcentrales hydroélectriques existantes.

\section{REMERCIEMENTS}

Ces études ont bénéficié de l'aide technique de J.M. Bach, D. Pujo, R. Galland, E. Galiay, D. Barracou et P. Laborie.

Nous remercions l'INRA de Saint Pée sur Nivelle pour avoir mis à notre disposition un bassin de stockage pour les smolts à la pisciculture de Lées-Athaas ainsi que EDF, en particulier le GEH d'Oloron et le groupement d'Asasp.

\section{BIBLIOGRAPHIE}

BAGLINIERE J.L., 1976. Etude des populations de Saumon atlantique (Salmo salar L., 1766) en Bretagne-Basse-Normandie. 1 - Caractéristiques des smolts de la rivière Ellé. Ann. Hydrobiol., 7, 141-158.

BOEUF G., 1994. La phase de préadaptation à la vie en mer : la smoltification. In Le saumon atlantique, ed. IFREMER (sous la direction de Gueguen J.C. et Prouzet P.), 47-63.

BOURGEOIS C.E., O'CONNELL M.F., 1988. Observations on the seaward migration of Atlantic salmon (Salmo salar L.) smolts through a large lake as determined by radiotelemetry and Carlin tagging studies. Can. J. Zool., 66, 685-691.

BOUSQUET B., 1979. Biologie et migration des smolts de saumon atlantique (Salmo salar L.) dans les bassins Loire-Allier et Adour-Gave d'Oloron. Thèse de Doctorat Inst. Nat. Polytech., Toulouse, France, $67 \mathrm{p}$.

CHORDA J., LARINIER M., 1997. Aménagement du canal d'amenée de Bedous. Etude sur modèle réduit. Rapport HYDRE n¹99-GHAAPPE 97.04, 6 p. 
CROZE O., CHANSEAU M., LARINIER M., 1999. Efficacité d'un exutoire de dévalaison pour smolts de saumon atlantique (Salmo salar L.) et comportement des poissons au niveau de l'aménagement hydroélectrique de Camon sur la Garonne. Bull. Fr. Pêche Piscic., 353/354, 121-140.

E.P.R.I., 1994. Research update of fish protection technologies for water intakes. EPRI TR-104122, $208 \mathrm{p}$.

FIELDS P.E., MURRAY A.K., JOHNSON D.E., FINGER G.L., 1958. Guiding migrant salmon by light repulsion and attraction in fast and turbid water. College of Fisheries, University of Washington, Technical Reports 36 and 41, 44 p.

GINETZ R.M., LARKIN P.A., 1976. Factors affecting rainbow trout (Salmo gairdneri) predation on migrant fry of sockeye salmon (Onchorynchus nerka). J. Fish. Res. Board Can., 33(1), 19-24.

GoSSET C., TRAVADE F., VOISIN M., GARAICOECHEA C., 1997. Etude de dispositifs de dévalaison pour smolts de saumon atlantique. Centrale hydroélectrique de Halsou (Nive - 64). Rapport de contrat INRA/EDF, 42 p.

gosset C., TRAVADE F., LABASTIE C., GARAICOECHEA C., 1998. Etude de dispositifs de dévalaison pour le saumon atlantique : influence de la lumière sur l'efficacité des exutoires de dévalaison. Centrale hydroélectrique de Halsou (Nive - 64). Rapport de contrat INRA/EDF, St Pée sur Nivelle, 34 p.

HANSEN L.P., JONSSON B., 1985. Downstream migration of hatchery-reared smolts of Atlantic salmon Salmo salar L. in the river Imsa, Norway. Aquaculture, 45, 237-248.

HANSEN L.P., JONSSON B., MORGAN R.I.G., THORPE J.E., 1989. Influence of parr maturity on emigration of smolting salmon (Salmo salar). Can. J. Fish. Aquat. Sci., 46, 410-415.

HAYMES G.T., PATRICK P.H., ONISTO L.J., 1984. Attraction of fish to mercury vapour light and its application in a generating station forebay. Int. Revue ges. Hydrobiol., 69, 867-876.

HOAR W.S., 1976. Smolt transformation : evolution, behavior and physiology. J. Fish. Res. Bd. Can., 33, 1234-1252.

KAWAMURA G., 1986. Vision and behaviour of fish in the vicinity of fish lamp. Dev. Man. Trop. Liv. Aquat. Res., Int. Conf., Serdang, Malaysia, 197-204.

KEMA N.V., 1982. Experimental reduction of fish impingement by artificial illumination at Bergum power station. Int. Revue ges. Hydrobiol., 67, 887-900.

KYNARD B.E., 1993. Anadromous fish behaviour important for fish passage. In : WILLIAMS E.P., SCRUTON D.A., GOOSNEY R.F., BOURGEOIS C.E., ORR D.C., RUGGLES C.P., (Eds.), Proc. Workshop on Fish Passage at Hydroelectric Developments, 1991, St. John's, Newfoundland, Can. Tech. Rep. Fish. Aquat. Sci., 1905, 95-105.

LARINIER M., DARTIGUELONGUE J., 1989. La circulation des poissons migrateurs : le transit à travers les turbines des installations hydroélectriques. Bull. Fr. Pêche Piscic., 312-313, 1-90.

LARINIER M., BOYER-BERNARD S., 1991a. Dévalaison des smolts et efficacité d'un exutoire de dévalaison à l'usine hydroélectrique d'Halsou sur la Nive. Bull. Fr. Pêche Piscic., 321, 72-92.

LARINIER M., BOYER-BERNARD S., 1991b. La dévalaison des smolts de saumon atlantique au barrage de Poutès sur l'Allier (43) : utilisation de lampes à vapeur de mercure en vue d'optimiser l'efficacité d'un exutoire de dévalaison. Bull. Fr. Pêche Piscic., 323, 129-148. 
LARINIER M., TRAVADE F., 1997. The development and evaluation of downstream bypasses for juvenile salmonids at small hydroelectric plants in France. American Fisheries Society, 127 ${ }^{\text {th }}$ Annual Meeting - Monterey, California, August 24-28 1997, $18 \mathrm{p}$.

MILLS D.H., 1989. Ecology and Management of Atlantic Salmon. Chapman and Hall, London, $351 \mathrm{p}$.

NEMETH R.S., ANDERSON J.J., 1992. Response of juvenile coho and chinook salmon to strobe and mercury vapor lights. N. Am. J. Fish. Manage., 12, 684-692.

OMBREDANE D., BAGLINIERE J.L., MARCHAND F., 1998. The effects of Passive Integrated Transponder tags on survival and growth of juvenile brown trout (Salmo trutta L.) and their use for studying movement in a small river. Hydrobiologia 371/372, 99-106.

PEAKE S., MCKINLEY R.S., SCRUTON D.A., MOCCIA R., 1997. Influence of transmitter attachment procedures on swimming performance of wild and hatchery-reared Atlantic salmon smolts. Trans. Am. Fish. Soc., 126, 707-714.

PITCHER T.J., 1993. Behaviour of Teleost Fishes. 2nd edition. Chapman and Hall, London, $715 \mathrm{p}$.

PORCHER J.P., TRAVADE F., 1992. Les dispositifs de franchissement : bases biologiques, limites et rappels réglementaires. Bull. Fr. Pêche Piscic., 326-327, $5-14$

PUCKETT K.J., ANDERSON J.J., 1988. Behavioral responses of juvenile salmonids to strobe and mercury lights. Univ. of Washington, Fish. Res. Inst., Tech. Rep. FRI-UW-8717, $35 \mathrm{p}$.

SMITH R.J.F., 1985. The control of fish migration. Zoophysiology vol. 17, Springer-Verlag, $243 \mathrm{p}$.

SOLOMON D.J., 1975. Observations on some factors influencing the migration of smolts of salmon Salmo salar L. and migratory trout Salmo trutta L. in a chalkstream. Int. Council Explor. Sea, M11, 1-10.

TAFT N., 1988. Evaluations of fish protection systems for use at hydroelectric plants. Hydro Review, 54-62.

THORPE J.F., 1981. Migration in salmonids with special reference to juvenile movements in freshwater. In : BRANNON E.L., SALO E.O., (Eds.), Salmon and trout migratory behavior symposium, 86-97.

THORPE J.E., MORGAN R.I.G., 1978. Periodicity in Atlantic salmon Salmo salar L. smolt migration. J. Fish Biol., 12, 541-548.

TRAVADE F., LARINIER M., 1992. La migration de dévalaison : problèmes et dispositifs. Bull. Fr. Pêche Piscic., 326-327, 165-176.

TRAVADE F., LARINIER M., INGENDAHL D., BACH J.M., PUJO D., BARRACOU D., 1996. Expérimentation d'un dispositif de dévalaison pour les juvéniles de saumon atlantique (année 1994). Usine hydroélectrique de Soeix (Gave d'Aspe - 64). Rapport GHAAPPE 96.02, 34 p.

TREMBLAY G., 1993. Etude de l'entraînement des saumonneaux dans le canal d'amenée de la centrale Mitis I en 1993. Rapport présenté à la vice-présidence Environnement Hydro-Québec, $41 \mathrm{p}$.

WOOD C.C., HARTGREAVES N.B., RUTHERFORD D.T., EMMETT B.T., 1993. Downstream and early marine migratory behavior of sockeye salmon Oncorhynchus nerka smolts entering Barkley Sound, Vancouver Island. Can. J. Fish. Aquat. Sci., 50, 1329-1337. 